\title{
Identification of UK sickness certification rates, standardised for age and sex
}

Gwenllian Wynne-Jones, Christian D Mallen, Sara Mottram, Chris J Main and Kate M Dunn

\begin{abstract}
Background

There is growing interest in tackling the perceived 'sick note' culture in the UK.

Aim

The aim of this paper was to report the rates of sickness certification in a UK population, using sick certification rates as a precursor to addressing fitness for work.

\section{Method}

Electronic records from all 14 practices included in the Keele GP Research Network were reviewed; all sickness certification records from 2005 were retrieved and corresponding consultation records were examined. Participants were 148176 patients registered during 2005, including 6398 patients who received at least one sickness certificate during the same year.

Results

The rate of sickness certification was 101.67 certificates per 1000 person years (95\% confidence interval $[\mathrm{Cl}]=100.13$ to 103.21 ). This rate was significantly higher in women, at 109.76 certificates per 1000 person years $(95 \% \mathrm{Cl}=107.550$ to 112.02$)$, compared to men who had a rate of 93.68 certificates per 1000 person years $(95 \% \mathrm{Cl}=91.59$ to 95.78 ; $P<0.001)$. The rate of sickness certification was greatest for mental health conditions, followed closely by musculoskeletal conditions.

\section{Conclusion}

On average, one in 10 patients will receive a sickness certificate each year, with the highest rates occurring around 50 years of age, in women. Mental health and musculoskeletal conditions were associated with the highest rates of certification. These results provide important information to underpin the national 'Fit for Work' scheme, by providing targets for intervention and a benchmark against which the impact of public health initiatives to reduce certified sickness absence due to health conditions can be evaluated and monitored.

Keywords

epidemiology; general practice; primary care; sickness certification.
\end{abstract}

\section{INTRODUCTION}

There is growing interest in tackling the perceived 'sick note' culture in the UK, with the government actively aiming to reduce the number of employees who are signed off sick each year. In 2005, a crossgovernment programme was launched to address the issues of Health, Work, and Wellbeing; ${ }^{1}$ the involvement of a wide range of partners demonstrates the importance placed on the programme. Despite this collaboration, costs of poor health to the workforce remain high; in 2007 the Confederation of British Industry (CBI) estimated that poor health in the workplace accounted for 175 million days lost, at a cost of $£ 13.4$ billion. $^{2}$

A review undertaken as part of the Health, Work, and Wellbeing programme, entitled Working for a Healthier Tomorrow, advocates the development of 'Fit for Work' services based on case-managed, multidisciplinary approaches. ${ }^{3}$ The review by Black recommends that the Fit for Work service be piloted and that the service be comprehensively evaluated. ${ }^{3}$ The accuracy of any such evaluation depends on the determination of appropriate base rates of sickness certificates in the community. Wide differences in the rates of sickness certification across Europe have been reported, demonstrating disparity among

G Wynne-Jones, RN(adult), BSc(Hons), PhD; CD Mallen, PhD, DROCG, DFFP, MRCGP; S Mottram, BSc(Hons), MSc; CJ Main, MA(Hons), MPhil, PhD, CClin Psychol; KM Dunn, BSc(Hons), MPhil, PhD, Arthritis Research Campaign, National Primary Care Centre, Primary Care Sciences, Keele University.

\section{Address for correspondence}

Gwenllian Wynne-Jones, Arthritis Research Campaign National Primary Care Centre, Primary Care Sciences, Keele University, Keele, Staffordshire, ST5 5BG.

E-mail: g.wynne-jones@cphc.keele.ac.uk

Submitted: 27 August 2008; Editor's response: 28 October 2008; final acceptance: 15 January 2009.

(c)British Journal of General Practice

This article was originally online first on 11 June 2009. Cite this article as: Br J Gen Pract 2009; 59: 510-516. Advance online publication. DOI: 10.3399/bjgp09X453431 
different cultures and healthcare systems, but there were no studies from the UK. ${ }^{4}$ Establishing UK rates of sickness certification would allow the development of a baseline against which interventions to reduce certification may be evaluated; furthermore, there is little UK research that has assessed which conditions result in sickness certification. ${ }^{5}$ Understanding which conditions account for the largest proportion of certificates would enable targeting of interventions, either within primary care or within the workplace.

In the UK, proxy measures are used to estimate rates of sickness certification based on work absence. The Chartered Institute of Personnel and Development (CIPD) reports the rate of UK sickness absence in terms of a percentage of lost working hours, ${ }^{6}$ estimating sickness absence as $3.5 \%$ of working hours. However, reporting rates of absence as a percentage of lost working hours does not reflect the clinical issues associated with certification in terms of the numbers of individuals absent from the workplace, nor does it represent the number of consultations for certification in general practice. ${ }^{7,8}$ The Department for Work and Pensions estimates that 4.48 million working-age people claimed key benefits in 2005, 3 million of whom were claiming sickness benefits. ${ }^{9}$ These estimates do not include those claiming statutory sick pay, therefore underestimating the true figure, and lacking any information on reasons for certification.

The Keele GP Research Partnership (KGPRP) holds frozen archive data on sickness certification in its Medical Certificates in Primary Care Archive and consultation data in its Consulters in Primary Care Archive, and therefore it offers an almost unrivalled opportunity to make this link.

\section{Objectives}

The objectives of this study were to report the rate of sickness certification in a UK population and identify the most common conditions for which sickness certificates are issued, and the rate of certification for these conditions.

\section{METHOD}

The KGPRP consists of 28 GP practices in North Staffordshire, 14 of which provide data for the sickness certification and consultation frozen archives. During 2005 these practices represented a GP population base, registered for the full year, of 148176 patients between the ages of 20 and 64 years. The datasets have been demonstrated to be valid in their recording of sickness certificates and consultation data respectively. ${ }^{10-12}$

All sickness certification records for 2005 were downloaded from the Medical Certificates database;

\section{How this fits in}

There is growing interest in tackling the perceived 'sick note' culture in the UK as demonstrated by the UK government's 'Fit for Work' initiative. The Fit for

Work initiative needs to be underpinned by current rates of sickness

certification, allowing the impact of such an initiative to be measured. This

study has demonstrated that, on average, one in 10 patients will receive a

sickness certificate each year, and there are trends in certification rate by age,

sex, and reason for certification.

records for working-age adults (aged 20-64 years) were eligible. Each sickness certification record included a unique individual identification number and practice identification number, plus patient age and sex, year of issue, date of issue, and the Read code used to identify the record of a sickness certificate. Read codes are a hierarchy of morbidity, symptom, and process codes, which become more specific further down the hierarchy. Sickness certificates are included as a process code and do not have a morbidity or symptom code attached; therefore, the corresponding consultation data are needed to identify the condition for which the certificate was issued. ${ }^{13}$ Read codes were used to identify the type of certificate - MED3, MED4, MED5, MED6, and private medical certificates. ${ }^{14}$

All consultation records for the year 2005 for individuals with sickness certificates (identified using the unique individual identification number) were downloaded from the consultation database. Each consultation record included the unique individual identification number and practice identification number, plus patient age and sex, and year of consultation, date of consultation, the Read code that was used to identify the problem with which the patient consulted, and the consultation free text.

Each sickness certification record was then matched to a consultation record on the same date using the unique individual identification number and the date of sickness certificate/consultation. To enable the analysis of conditions for which certificates are issued, the Read codes from the consultation data were used to identify broad categories; these categories corresponded with the chapter level of the Read code system. ${ }^{13}$ Those consultations that could not be coded using this method, that is no Read code was provided or the Read code did not match the standard format, were coded manually by reviewing the free text of the consultation. When reviewing the free text, if the reason for certification was not clear the certificate was coded as 'unknown reason'. Free text was initially reviewed, checked, and recoded; a total of 1504 certificates were coded manually $-10 \%$ of the 
total number of certificates issued. Perinatal conditions were excluded from analysis, as this chapter heading relates to patients up to 6 months of age. III-defined conditions and working diagnoses refer to a consultation where a definite diagnosis cannot be established; it can be used to record the symptoms the patient presents with, for example blackouts of unknown cause. A category was added to identify certificates that were post-operative; this was because there were a number of consultation records with no specific condition attached to the post-operative statement.

\section{Statistical methods}

Results are reported as recommended in a recent review of rates of sickness certification. ${ }^{4}$ The rate of certification was defined as the number of certificates issued, divided by the number of registered patients in 2005 . The crude rate of certification in the total population, and rates stratified by age group and sex were calculated, with 95\% confidence intervals (Cls); a $t$-test for differences in proportions was also calculated. Rates are presented per 1000 person years. Only patients

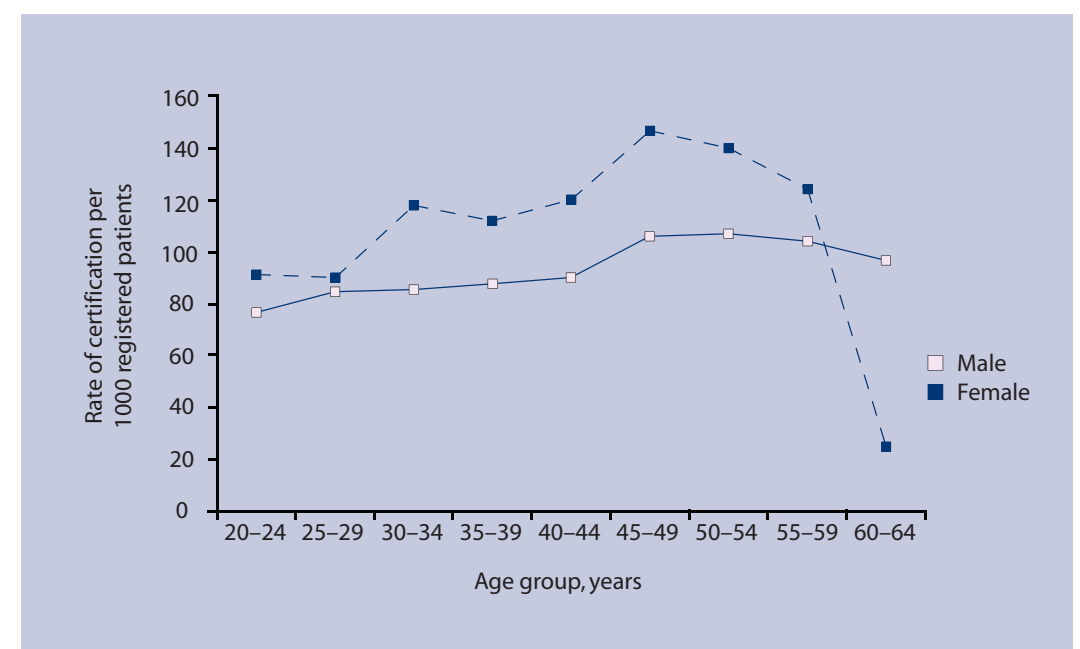

registered for the whole year were included in these analyses.

Certification rates for health conditions defined by Read code chapters (Figure 1) were calculated with 95\% Cls. The total, and age- and sex-specific rates were then directly age standardised to the UK 2005 population. ${ }^{15}$ All analysis was carried out using SPSS (version 14.0).

\section{RESULTS}

\section{Rates of sicknesses certification by age and sex}

During the year 2005, 148176 patients were registered at the 14 GP practices; 6398 patients received one or more sickness certificates and were included in the analysis. This gives a prevalence of sickness certification of $4 \%$. These individuals received a total of 15640 certificates ranging from 1 to 20 certificates per person during the year 2005, an average of 2.44 certificates per person. A total of 15065 certificates could be coded with a health condition and were included in the condition-specific analysis; $4 \%$ of certificates that could not be coded with a reason for certification were excluded.

The overall rate of sickness certification was 101.67 certificates per 1000 person years $(95 \% \mathrm{Cl}=$ 100.13 to 103.21$)$. The rate of sickness certification was statistically significantly higher in women, at 109.76 certificates per 1000 person years $(95 \% \mathrm{Cl}=$ 107.55 to 112.02 ), compared to men who had a rate of 93.68 certificates per 1000 person years $(95 \% \mathrm{Cl}$ $=91.59$ to 95.78$)(P<0.001)$. The rate of sickness certification increased with age, peaking in the 45-49 year age group and falling off slightly before retirement (Table 1 and Figure 1).

\section{Standardised rates}

Standardising the rate of certification to the UK population results in a similar rate of certification overall at $101.15(95 \% \mathrm{Cl}=101.05$ to 101.26$)$
Figure 1. Crude rate of sickness certification by age and sex per 1000 registered patients.
Table 1. Rates of sickness certification by age and sex.

\begin{tabular}{|c|c|c|c|c|c|}
\hline \multirow{2}{*}{$\begin{array}{l}\text { Age, } \\
\text { years }\end{array}$} & \multirow{2}{*}{$\begin{array}{l}\text { Total number of } \\
\text { certificates issued }\end{array}$} & \multirow{2}{*}{$\begin{array}{l}\text { Registered } \\
\text { population }\end{array}$} & \multicolumn{3}{|c|}{ Sickness certification rate per 1000 person years $(95 \% \mathrm{Cl}$ ) } \\
\hline & & & Total & Males & Females \\
\hline $20-24$ & 1136 & 13534 & 83.94 (79.26 to 88.61$)$ & 76.92 (70.63 to 83.22$)$ & 91.18 (84.27 to 98.10$)$ \\
\hline $25-29$ & 1156 & 13266 & 87.14 (82.34 to 91.94$)$ & 84.47 (77.81 to 91.12$)$ & 89.87 (82.95 to 96.79 ) \\
\hline $30-34$ & 1670 & 16384 & 101.93 (97.3 to 106.56$)$ & 85.82 (79.75 to 91.88$)$ & 118.04 (111.05 to 125.03$)$ \\
\hline $35-39$ & 1957 & 19624 & 99.72 (95.53 to 103.92 ) & 87.84 (82.26 to 93.422$)$ & 111.81 (105.55 to 118.07$)$ \\
\hline 40-44 & 2046 & 19537 & $104.72(100.43$ to 109.02$)$ & 90.05 (84.39 to 95.71$)$ & 119.61 (133.15 to 126.07$)$ \\
\hline $45-49$ & 2190 & 17329 & 126.38 (121.43 to 131.33$)$ & 106.07 (99.61 to 112.54$)$ & $146.90(139.43$ to 154.38$)$ \\
\hline $50-54$ & 1975 & 16029 & 123.21 (118.13 to 128.30$)$ & 107.33 (100.62 to 114.03$)$ & ) 139.81 (132.14 to 147.49$)$ \\
\hline $55-59$ & 2053 & 17968 & 114.26 (109.61 to 118.91$)$ & 104.23 (97.88 to 110.58$)$ & 124.09 (117.31 to 130.87$)$ \\
\hline $60-64$ & 882 & 14505 & 60.81 (56.92 to 64.70$)$ & 96.67 (89.87 to 103.46$)$ & 24.85 (21.27 to 28.44$)$ \\
\hline All ages & 15065 & 148176 & $101.67(100.13$ to 103.21$)$ & 93.68 (91.59 to 95.78$)$ & 109.76 (107.50 to 112.02$)$ \\
\hline
\end{tabular}


certificates per 1000 person years. The trends by age and sex persist in the standardised rates, with women having a higher rate of certification at 109.53 $(95 \% \mathrm{Cl}=109.37$ to 109.68$)$ per 1000 person years compared to men at a rate of $92.85(95 \% \mathrm{Cl}=92.70$ to 92.99$)$ certificates per 1000 person years.

\section{Rates of sicknesses certification by reason for consultation}

The condition-specific sickness certification rates are shown in Table 2. The rates for five Read code chapters were above 5 certificates per 1000 person years: mental disorders, respiratory system, musculoskeletal disorders, ill-defined/working conditions, and injury/poisoning. The age- and sexrelated rates of sickness certification for the three most common specific health conditions, mental health, musculoskeletal system, and respiratory system, are shown in Figure 2. The highest rate is for mental health conditions in women, which reached a peak of 43.37 certificates per 1000 person years $(95 \% \mathrm{Cl}=38.86$ to 47.88$)$ at the $50-54$ year age group, then fell off rapidly towards retirement age. By contrast, mental health conditions in men peaked at 25.97 certificates per 1000 person years $(95 \% \mathrm{Cl}=$ 22.16 to 29.77 ) in the 25-29 year age group, with the rate gradually falling with age after that. The patterns among men and women were largely similar for both musculoskeletal and respiratory problems, although the rates were higher in men for musculoskeletal problems, and higher in women for respiratory problems. The rates of musculoskeletal problems increased with age, peaking in men at 45-49 years with a rate of 33.75 certificates per 1000 person years $(95 \% \mathrm{Cl}=29.96$ to 37.54$)$, and peaking in women at the 55-59 year age group, with a rate of 32.07 certificates per 1000 person years $(95 \% \mathrm{Cl}=28.44$ to 35.69). The rates of respiratory conditions did not vary much across the age groups, although there was a peak at 50-54 years at 9.65 certificates per 1000 person years in males $(95 \% \mathrm{Cl}=7.53$ to 11.76$)$, and 12.25 certificates per 1000 person years in females $(95 \% \mathrm{Cl}=9.81$ to 14.68$)$ (Table 2 and Figure 2).

\section{DISCUSSION}

\section{Summary of main findings}

This study demonstrated that, on average, one in 10 patients registered at the 14 practices included in the Keele GP Research Partnership received a sickness certificate during the 1-year study period. The rate increased with age until around 50 years, and the overall rate of certification was higher in women compared to men up to the age of 60 years. When standardising these rates to the UK population, the rates remained similar with an estimated one in 10 of the adult population receiving a sickness certificate.
Table 2. Number and rates of sickness certificates issued by reason for consultation.

Total number of Sickness certification rate per Read code chapter heading certificates issued 1000 person years $(95 \% \mathrm{Cl})$, total ${ }^{\mathrm{a}}$

\begin{tabular}{|c|c|c|}
\hline Infectious/parasitic diseases & 365 & 2.46 (2.21 to 2.72$)$ \\
\hline Neoplasms & 219 & $1.48(1.28$ to 1.67$)$ \\
\hline Endocrine/metabolic & 159 & 1.07 (0.91 to 1.24$)$ \\
\hline Blood diseases & 24 & $0.16(0.10$ to 0.23$)$ \\
\hline Mental disorders & 4116 & 27.78 (26.94 to 28.68$)$ \\
\hline Nervous system & 653 & 4.41 (4.07 to 4.47 ) \\
\hline Circulatory system & 591 & 3.99 (3.67 to 4.31$)$ \\
\hline Respiratory system & 1053 & 7.11 (6.68 to 7.53$)$ \\
\hline Digestive system & 676 & 4.56 (4.22 to 4.91$)$ \\
\hline Genitourinary system & 474 & 3.20 (2.91 to 3.49$)$ \\
\hline Pregnancy/childbirth/puerperium & 324 & 2.19 (1.95 to 2.42$)$ \\
\hline Skin/subcutaneous tissue & 397 & $2.68(2.42$ to 2.94$)$ \\
\hline Musculoskeletal & 3384 & 22.84 (22.08 to 23.60$)$ \\
\hline Congenital anomalies & 13 & 0.09 (0.04 to 0.14$)$ \\
\hline $\begin{array}{l}\text { III-defined conditions/ } \\
\text { working diagnoses }\end{array}$ & 983 & $6.63(6.22$ to 7.05$)$ \\
\hline Injury/poisoning & 1154 & 7.79 (7.34 to 8.24$)$ \\
\hline Causes of injury/poisoning & 122 & $0.82(0.68$ to 0.97$)$ \\
\hline Post-operative $^{b}$ & 325 & 2.19 (1.96 to 2.43$)$ \\
\hline Unknown reason ${ }^{c}$ & 595 & 4.02 (3.69 to 4.34$)$ \\
\hline
\end{tabular}

${ }^{a}$ Rates are not presented by sex, as the numbers are small. ${ }^{b}$ Not a Read code label, but included as many certificates were labelled as post-operative without a condition attached. "Not a Read code label.

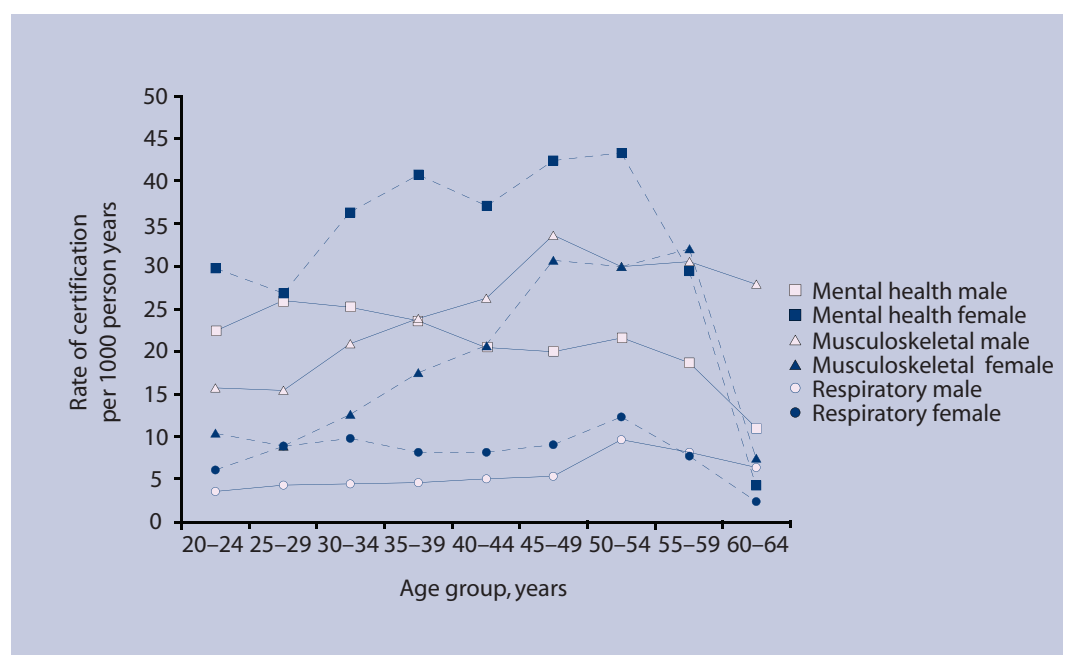

The highest rate of certification for a specific health condition as defined by Read code 'chapters' was mental health conditions, followed by musculoskeletal Figure 2. Rates of sickness certification by the three most common conditions. conditions, injury, and respiratory conditions.

\section{Strengths and limitations of the study}

There are a number of limitations to this study. First, it was not possible to capture the duration of sickness certificates due to the limited amount of data that is recorded within the sickness certificate record. It could be that the observed certification rate 
of 100 per 1000 person years represents a certificate of 100 days or 100 weeks, depending upon the duration of the certificate. The most frequent duration of certification reported in the literature is approximately 2 weeks, ${ }^{16-19}$ and there is little reason to believe that the GPs in the current study would differ to any great degree. The duration of certification may be affected by a number of other issues such as sex, age, or condition. The literature presents evidence of no difference in certification by sex, but there is some increase in duration with increasing age. ${ }^{17,19-21}$ There is also evidence that duration of certification is associated with condition; in particular, musculoskeletal and mental health conditions have longer certified absences. ${ }^{19,21}$ The lack of information on duration in this paper could mean that total length of certification for musculoskeletal and mental health conditions is actually higher than indicated by the rates reported here, as not only are the rates higher but the duration could also be longer. There is no evidence that the duration of certification has changed over time or that duration would be influenced by the implementation of the Fit for Work initiative; therefore, the rates may provide a useful baseline from which to measure the overall number of certificates that are being issued. In applying the sickness certification rates in this paper to the general population, generalisability should be considered. The proportions of males and females in each age category were similar to the standard UK population (data not shown); ${ }^{15,22,23}$ this is supported by the standardised data, which varied little from crude rates. North Staffordshire has a higher proportion of lower socioeconomic classes compared to national levels, ${ }^{15,22,23}$ and self-reported sickness absence has been demonstrated to be increased in lower socioeconomic classes, ${ }^{24}$ so it is possible that North Staffordshire rates could be slightly higher than a true national rate.

The study also has a number of strengths. The results are derived from a large dataset of sickness certification records linked to consultation data, allowing the rate and patterns of certification to be calculated by age, sex, and specific condition. This is the first study to assess the rate of sickness certification in a UK population; previous work reports rates of new incapacity benefits as a proxy for the rate of sickness certificates. ${ }^{25-27}$ However, using incapacity benefit data underestimates the rate of sickness certification, as only those who are certified for 28 weeks are eligible to apply for this benefit. ${ }^{12}$

\section{Comparison with existing literature}

The specific conditions that have increased rates of sickness certificates match with the most frequent conditions seen in occupational health, that is, musculoskeletal disorders and psychiatric problems. ${ }^{28-30}$ European studies have also reported musculoskeletal and mental health conditions as the most common reasons for certification. ${ }^{31-34}$ It is possible that the rate of certification is higher in some conditions as a result of increased primary care attendance. For example, the rate of certification for mental health conditions, musculoskeletal conditions, injury, and respiratory conditions may be increased as they are all associated with frequent attendance in general practice. ${ }^{35,36}$ The reasons for frequent attendance are varied and it has been suggested that there is a complex relationship between morbidity and individual patient characteristics which determines who consults their GP. These conditions may have a greater impact on individuals, including their ability to work, and increased rates of certification are purely a function of the condition severity.

\section{Implications for future research and practice}

The results of the current paper may provide a benchmark against which the impact of public health initiatives to reduce certified sickness absence due to health conditions may be evaluated and monitored. This paper has also identified specific subgroups where sickness certification is increased and that could be targeted in interventions aimed at reducing certification.

\section{Funding body}

Christian Mallen is funded by an Arthritis Research Campaign Primary Care Fellowship (18271)

\section{Ethical approval}

Approval for use of the Medical Certificates and Consultation databases was gained from the North Staffordshire Research Ethics Committee (LREC Project 03/04)

\section{Competing interests}

The authors have stated that there are none

\section{Acknowledgements}

We would like to thank the Keele GP Research Partnership, and Ian Thomas and Simon Wathall for their assistance with data retrieval.

\section{Discuss this article}

Contribute and read comments about this article on the Discussion Forum: http://www.rcgp.org.uk/bjgp-discuss

\section{REFERENCES}

1. Health, Work, Wellbeing. Working for health. http://www. workingforhealth.gov.uk/Default.aspx (accessed 28 Apr 2009).

2. CBI, AXA. Workplace absence rises amid concerns over long-term sickness - CBI/AXA survey. CBI News Release, 2007. http://www. cbi.org.uk/ndbs/press.nsf/0363clf07c6ca12a8025671c00381cc7/1032fea 0526c09df802572b3003ea789? OpenDocument (accessed 21 May 2009).

3. Black C. Working for a healthier tomorrow. London: The Stationery Office, 2008.

4. Wynne-Jones G, Mallen CD, Welsh V, Dunn KM. Rates of sickness certification in European primary care: a systematic review. Eur J Gen pract 2009; 3: 99-108.

5. Bradshaw SE. Life's work: occupational health - the wealth of the nation. Br J Gen Pract 2008; 58(554): 605-606. 


\section{COMMENTARY}

\section{From sickness to fitness: modernising medical certification}

The coming years herald a radical overhaul of the sickness absence certification process in the UK, under direction of the Department of Work and Pensions (DWP). Decreased productivity, days lost and the associated healthcare expenses of ill health in people of working age in the UK is estimated to cost in excess of $£ 100$ billion per annum. 'This sum is equivalent to the gross domestic product of Portugal, and is greater than the total annual running costs of the NHS. ${ }^{2}$ It is an accepted view that work can be beneficial to health. Therefore there is a move towards promoting able employees back into the workplace. This not only improves the individuals' socioeconomic wellbeing, but also aims to reduce the financial burden imposed upon society.

GPs are typically the first healthcare professionals consulted by patients who are absent from work through illness. It is recognised that the recommendations and support GPs communicate to patients and their employers via 'sick notes' is an indispensable form of medical therapy to both parties. Sick notes are there principally to allow employees time and allowance for necessary medical recuperation, but the information provided can also enable organisations to think ahead and plan their workforce in the most costeffective way.

Current sick notes, such as the 'Med-3', have remained largely unchanged for over half a century, but they have limitations. They centre on the causes of illness, rather than the consequences, and oblige doctors to impose rather rigid and arbitrary periods of sick leave. Confirming to an employer that their employee is suffering from ill health is very different to making a judgement on whether or not they are well enough to do all or some aspects of their job, and the existing system does little to assist doctors in making that decision.

Improving Health and Work: Changing Lives, ${ }^{3}$ published in November 2008, was the Government's response to Working for a Healthier Tomorrow, 'Dame Carol Black's review of the health of Britain's working age population. The key message from this publication of relevance to general practice is that the DWP has now formulated several new strategies for sick certification and in managing return to work after sick leave. ${ }^{3.4}$ These reforms include a new 'fit note' programme for GPs to improve knowledge and skills in occupational medicine.

The fit note system ${ }^{3}$ switches the focus of doctors' advice to what patients can still manage to do rather than what they can't do. This is designed to assist patients and employers in considering whether, with modified tasks and workplace adaptation, a patient's earlier return to work might be accommodated where reasonable. So far, paper and electronic versions have been tested with over 500 GPs from across the UK. This, and other 'Fit for Work' schemes, developed with the support of healthcare professionals, trade unions, and employer representatives, will roll out across Britain in spring 2010.

GPs will be at the forefront of these changes and will have a pivotal role as both the gatekeepers and evaluators of this new sick certification system.

\section{Steven E Bradshaw,}

Oxford Radcliffe Hospitals NHS Trust. E-mail: stevenb117@hotmail.co.uk

\section{Provenance}

Commissioned; not peer reviewed.

\section{REFERENCES}

1. Black C. Working for a healthier tomorrow. London: TSO, 2008. http://www.workingforhealth.gov.uk/documents/working-for-a-healthier-tomorrow-tagged.pdf (accessed 27 May 2009).

2. Bradshaw SE. Life's work: occupational health — the wealth of the nation. Br J Gen Pract 2008; 58(554): 605-606.

3. The Stationery Office. Improving health and work: changing lives. London: TSO, November 2008. http://www.workingforhealth.gov.uk/documents/large-print-improvinghealth-and-work-changing-lives.pdf (accessed 27 May 2009).

4. Department for Work and Pensions. 25 November 2008 - Keep Britain working — Johnson and Purnell respond to Dame Carol Black report. http://www.dwp.gov.uk/mediacentre/pressreleases/2008/nov/emp118-251108.asp (accessed 2 Jun 2009).

DOI: 10.3399/bjgp09X453549

6. Chartered Institute of Personnel and Development. Absence management: a survey of policy and practice. London: Chartered Institute of Personnel and Development, 2005

7. Hiscock J, Ritchie J. The role of GPs in sickness certification. Report No 148. Leeds: HMSO, 2001.

8. Hussey S, Hoddinott P, Wilson P, et al. Sickness certification system in the United Kingdom: qualitative study of views of general practitioners in Scotland. BMJ 2004; 328(7431): 88.

9. Department for Work and Pensions. Client group analysis: quarterly report on the population of working age on key benefits - February 2005. DWP: London, 2005.

http://www.dwp.gov.uk/asd/asd1/cga_wa/CGA_WA_Feb05_bulletin.pd f (accessed 26 May 2009).

10. Porcheret M, Hughes R, Evans D, et al. Data quality of general practice electronic health records: the impact of a program of assessments, feedback, and training. J Am Med Inform Assoc 2004; 11(1): 78-86.

11. Jordan K, Clarke AM, Symmons DP, et al. Measuring disease prevalence: a comparison of musculoskeletal disease using four general practice consultation databases. Br J Gen Pract 2007; 57(534): 7-14.

12. Wynne-Jones G, Mallen CD, Main CJ, Dunn KM. Sickness certification in general practice: a comparison of electronic records with selfreported work absence. Primary Healthcare Research and Development 2008; 9: 113-118. doi:10.1017/S1463423608000650.

13. NHS Information Authority. The clinical terms version 3 (the Read 
codes). Birmingham: NHS Information Authority, 2000.

14. Hiscock J, Ritchie J, National Centre for Social Research, Department for Work and Pensions. The role of GPs in sickness certification; a report of research carried out by the National Centre for Social Research on behalf of the Department for Work and Pensions. Report No 148. Leeds: Department for Work and Pensions, 2001.

15. Office for National Statistics. Neighbourhood statistics, 2001. Newport: Office for National Statistics, 2008.

16. Head J, Ferrie JE, Alexanderson K, et al. Diagnosis-specific sickness absence as a predictor of mortality: the Whitehall II prospective cohort study. BMJ 2008; 337: a1469.

17. Tellnes G. Days lost by sickness certification. Scand J Prim Health Care 1989; 7(4): 245-251.

18. Reiso H, Gulbrandsen P, Brage S. Doctors' prediction of certified sickness absence. Fam Pract 2004; 21(2): 192-198.

19. Bollag U, Rajeswaran A, Ruffieuxf C, Burnand B. Sickness certification in primary care - the physician's role. Swiss Med Wkly 2007; 137(23-24): 341-346.

20. Dünner S, Decrey H, Burnand B, Pecoud A. Sickness certification in primary care. Soz Praventivmed 2001; 46(6): 389-395.

21. Tellnes G. Duration of episodes of sickness certification. Scand J Prim Health Care 1989; 7(4): 237-244.

22. Office for National Statistics. Standard occupational classification. Vol. 2. The coding index. London: The Stationery Office, 2000.

23. Office for National Statistics. The National Statistics socio-economic classification user manual. Version 1.1. London: Office for National Statistics, 2002.

24. Benavides FG, Benach J, Mira M, et al. Occupational categories and sickness absence certified as attributable to common diseases. Eur $J$ Public Health 2003; 13(1): 51-55.

25. Waddell G, Aylward M. The scientific and conceptual basis of incapacity benefits. London: The Stationery Office, 2005

26. Kemp PA, Davidson J. Routes onto incapacity benefit: findings from a survey of recent claimants. Report No 469. Leeds: Department for Work and Pensions, 2007.

27. Shiels C, Gabbay MB, Ford FM. Patient factors associated with duration of certified sickness absence and transition to long-term incapacity. $\mathrm{Br}$ Gen Pract 2004; 54(499): 86-91.

28. Department for Work and Pensions. Incapacity benefit and severe disablement allowance quarterly summary statistics; May 2004. London: Department for Work and Pensions, 2004.

29. Smedley J, Dick F, Sadhra S.Oxford handbook of occupational health. Oxford: Oxford University Press, 2007.

30. Hussey L, Turner S, Thorley K, et al. Work-related ill health in general practice, as reported to a UK-wide surveillance scheme. Br J Gen Pract 2008; 58(554): 637-640.

31. Shiels C, Gabbay M. The influence of GP and patient gender interaction on the duration of certified sickness absence. Fam Pract 2006; 23(2): 246-252.

32. Arrelov B, Borgquist L, Ljungberg D, Svardsudd K. The influence of change of legislation concerning sickness absence on physicians' performance as certifiers. A population-based study. Health Policy 2003; 63(3): 259-268

33. Englund L, Tibblin G, Svardsudd K. Effects on physicians' sick-listing practice of an administrative reform narrowing sick-listing benefits. Scand J Prim Health Care 2000; 18(4): 215-219.

34. Englund L, Svardsudd K. Sick-listing habits among general practitioners in a Swedish county. Scand J Prim Health Care 2000; 18(2): 81-86.

35. Foster A, Jordan $\mathrm{K}$, Croft P. Is frequent attendance in primary care disease-specific? Fam Pract 2006; 23(4): 444-452.

36. Vedsted P, Christensen MB. Frequent attenders in general practice care: a literature review with special reference to methodological considerations. Public Health 2005; 119(2): 118-137. 